\title{
Chapter 12 \\ Emerging Regenerative Approaches for Periodontal Regeneration: The Future Perspective of Cytokine Therapy and Stem Cell Therapy
}

\author{
Shinya Murakami
}

\begin{abstract}
Cytokine therapy using basic fibroblast growth factor (FGF-2) has attracted attention as a next-generation periodontal tissue regenerative therapy. Clinical trial studies to date have shown that local application of $0.3 \%$ FGF-2 induces statistically significant new alveolar bone formation. In vitro analyses using cultured periodontal ligament cells showed that FGF-2 maintains stem cells in an undifferentiated state and promotes the proliferation of these stem cells during the initial stages of wound healing. This increases the cell density of periodontal tissue stem cells at the site of healing, promotes angiogenesis, and produces specific extracellular matrix molecules, thereby preparing a local environment suitable for the regeneration of periodontal tissue. Additionally, based on an analysis in beagles, when adipose tissue-derived multi-lineage progenitor cells (ADMPCs) isolated from adipose tissue were transplanted together with fibrin gel to areas of periodontal tissue loss, significant regeneration of periodontal tissue was observed at the transplantation site. These results strongly suggest that adipose tissue, which is abundant in the human body and can be easily and safely collected, is a promising source of stem cells to promote the regeneration of periodontal tissue.
\end{abstract}

Keywords Periodontal regeneration $\bullet$ FGF-2 $\bullet$ ADSC

\footnotetext{
S. Murakami $(\bowtie)$

Department of Periodontology, Osaka University Graduate School of Dentistry,

1-8 Yamadaoka, Suita, Osaka 565-0871, Japan

e-mail: ipshinya@dent.osaka-u.ac.jp
} 


\subsection{Introduction}

Periodontal disease occurs when a bacterial biofilm (dental plaque) adheres to the boundary between the teeth and gingiva, causing chronic inflammation and progressively destroying the periodontal tissue that supports the teeth. Therefore, periodontal treatment involves scaling and root planing, which mechanically removes the causative bacteria biofilm together with the necrotic cementum from the surface of the tooth root. Appropriate application of this therapy eliminates periodontal tissue inflammation and stops the process of destruction of the same tissue. However, removing the cause of the disease does not regenerate the lost periodontal tissue to its original state. Given the high prevalence of periodontal disease both in Japan and worldwide and the need to maintain or enhance "QOL supported by the teeth and the mouth" in middle-aged and elderly people, developing highly predictable periodontal tissue regenerative therapy that can be performed as a follow-on after treatment to remove the cause is urgently needed.

\subsection{Periodontal Ligament as a Storage Site for Periodontal Tissue Stem Cells}

Induction of tissue regeneration requires the presence of stem cells. Studies of regenerative medicine for periodontal tissue have shown that tissue stem cells that make the regeneration of periodontal tissue possible are present in the tissue surrounding the tooth root, known as the periodontal ligament, even in adults. The periodontal ligament is a ligament tissue located between the cementum and alveolar bone, with a thickness of 100-200 $\mu \mathrm{m}$. Type I collagen fiber bundles form the main body of the periodontal ligament; the principal cellular components of this tissue include the periodontal ligament fibroblasts as well as characteristic cell groups such as osteoblasts and osteoclasts on the surface of alveolar bone and cementoblasts found on the cementum surface. Interestingly, the results of various molecular biological analyses of periodontal ligament tissue show that many of the cells in the periodontal ligament show consistently high expression of molecules such as RUNX-2 and alkaline phosphatase which are closely associated with cytodifferentiation into hard tissue-forming cells. These results suggest that the periodontal ligament is involved in remodeling hard tissues such as alveolar bone and cementum depending on environmental changes. Furthermore, undifferentiated mesenchymal stem cells, which can differentiate into a variety of cell types other than osteoblasts and cementoblasts, are present in the human periodontal ligament $[1,2]$. 


\subsection{Concept and Current Status of Periodontal Tissue Regenerative Therapy}

Although mesenchymal stem cells that can differentiate into osteoblasts and cementoblasts are present in the adult periodontal ligament, periodontal tissue does not successfully regenerate when conventional periodontal treatment is performed to remove the cause of periodontal disease. Thus, in addition to conventional treatment, it is necessary to develop a method for inducing the periodontal tissue stem cells present in the periodontal ligament.

The specific biological processes required for the regeneration of periodontal tissue can be summarized into the following three processes: (1) selective and preferential induction of periodontal ligament-derived cells on the tooth root surface facing the areas of periodontal tissue loss, (2) proliferation and migration of undifferentiated mesenchymal stem cells (periodontal tissue stem cells) contained within the periodontal ligament-derived cells while retaining their differentiation potential and then achieving site-specific differentiation as hard tissue-forming cells (osteoblasts and cementoblasts) and periodontal ligament fibroblasts, and (3) collagen fiber bundles produced by periodontal ligament fibroblasts becoming embedded into bone tissue and cementum newly formed by osteoblasts and cementoblasts, resulting in regeneration of the fibrous connections between the teeth and alveolar bone (Fig. 12.1).

\section{Cellular and molecular basis of periodontal tissue regeneration}

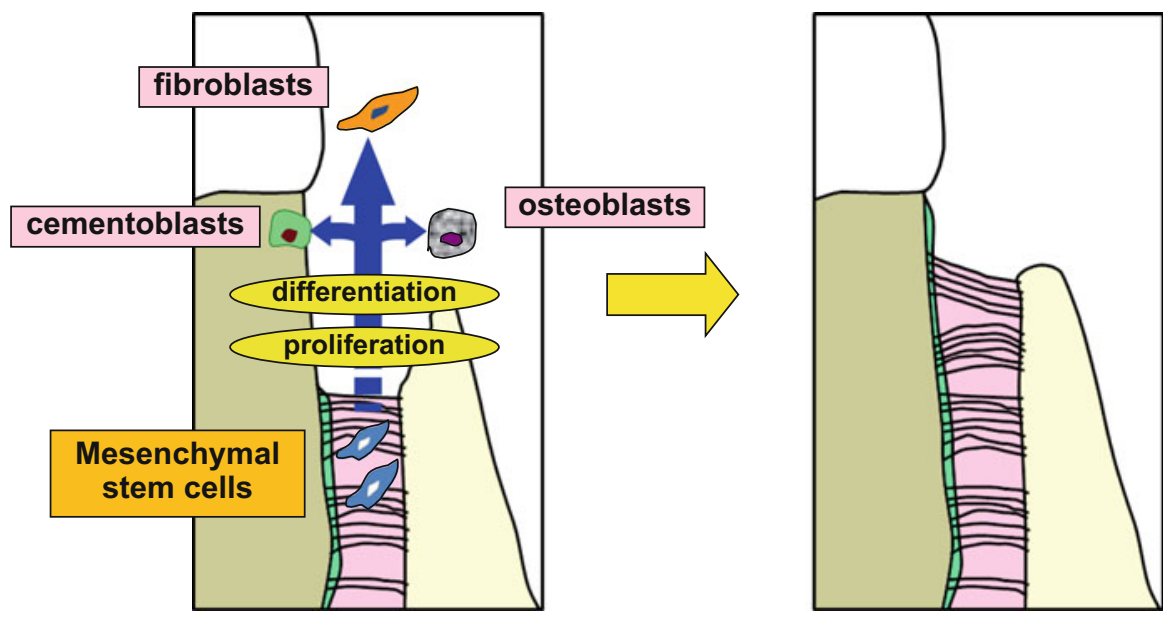

Fig. 12.1 Cellular and molecular basis of periodontal tissue regeneration. Periodontal tissue stem cells, including undifferentiated mesenchymal stem cells, are present in the periodontal ligament, and regeneration of periodontal tissue can be achieved by activating these cells to proliferate, migrate, and site specifically differentiate at the site of periodontal tissue loss 
Periodontal tissue regenerative therapies with clinical applications have been developed previously, including the "guided tissue regeneration method" and "enamel matrix protein method." These treatment methods have a long history of use in dental practice and have shown some degree of success. However, there are a number of issues remaining related to indicated conditions and predictability, and improvements to these methods are required. Therefore, the action mechanism should be determined and next-generation periodontal tissue regenerative therapy with stable predictability should be established.

\subsection{Possibility of Cytokine Therapy}

Human recombinant cytokines are currently used as therapeutic agents in a variety of diseases. In the field of periodontal tissue regenerative medicine, there have been many attempts to induce regeneration of periodontal tissue through the migration of periodontal tissue stem cells to the site of periodontal tissue loss and proliferation of these cells in the same area or differentiation to osteoblasts and cementoblasts, which are activated through local application of specific cytokines.

In the USA, a combination of platelet-derived growth factor (PDGF) and $\beta$-tricalcium phosphate $(\beta$-TCP) $(\beta$-TCP $+0.3 \mathrm{mg} / \mathrm{mL}$ PDGF) has been approved by the US Food and Drug Administration (FDA) to induce regeneration of periodontal tissue, which is marketed as GEM21S®. Cytokines are also applied in dentistry using a combination of bone morphogenic protein-2 (BMP-2) and bovine type I collagen, which has also been approved by the US FDA as a medical device used in alveolar ridge augmentation procedures and sinus elevation surgery. Thus, human recombinant cytokines are beginning to be applied in the field of dentistry.

\subsection{Inducing Periodontal Tissue Regeneration with Basic Fibroblast Growth Factor (FGF-2)}

Fibroblast growth factors (FGFs) are a group of proteins that promote fibroblast proliferation and were discovered in the brain and pituitary gland tissue. This family of proteins comprises FGF-1 to FGF-23. Among these, FGF-2 induces the proliferation of a wide variety of cells, including fibroblasts, vascular endothelial cells, neuroectodermal system cells, osteoblasts, chondrocytes, vascular smooth muscle cells, and epithelial cells. FGF-2 has attracted attention in the field of regenerative medicine because: (1) FGF-2 has potent angiogenesis-promoting action and (2) FGF-2 promotes the cellular proliferation of undifferentiated mesenchymal cells while retaining their pluripotency. An example of the clinical application of FGF-2 is as a therapeutic agent for intractable skin ulcers, such as decubital ulcers, and this therapy has been approved for manufacture in Japan. 
In our laboratory, we verified the efficacy and safety and determined whether FGF-2 promotes the regeneration of periodontal tissue in animal studies $[3,4]$. We experimentally prepared class II furcation involvement at the furcation sites of mandibular molars in beagles and nonhuman primate and filled the bone defect, on the experimental side, with $0.1-0.4 \%$ FGF- 2 with a gelatinous carrier and measured the histological morphology at 6 and 8 weeks after FGF-2 administration. The results showed that regeneration of periodontal tissue was associated with a statistically significant increase in new bone volume, new trabecular bone volume, and new cementum volume and was induced by local administration of FGF-2 (Fig. 12.2). At the same site, we observed the reappearance of Sharpey's fibers and rebuilding of fibrous attachments [3]. Extension of the peripheral nerves and Ruffini nerve endings was detected in the regenerated periodontal ligament. This strongly suggests that local administration of FGF-2 not only constructs periodontal tissue but also regenerates the function of sensory receptors in the tissue.

Furthermore, abnormal healing findings such as downgrowth of the gingival epithelium, ankylosis, and root resorption were not detected in cases on the side administered FGF-2.

We conducted an early phase II (PIIa) clinical trial (double-blind study with dose-response concurrent control, including placebo) with 13 participating facilities throughout Japan to investigate whether FGF-2 could induce the regeneration of periodontal tissue as well as the safety of FGF-2 (2002-2004). In this study, we investigated the efficacy and safety of FGF-2 as a drug to induce the regeneration of periodontal tissue using a placebo (3\% hydroxypropylcellulose, which was used as the carrier) and the investigational drug containing $0.03 \%, 0.1 \%$, and $0.3 \%$ FGF-2. The results revealed a statistically significant induction of new alveolar bone formation based on standardized x-ray images after local administration of $0.3 \%$ FGF-2 to human 2- or 3-wall intrabony defects in the alveolar bone [5]. Next, we implemented a late phase II (PIIb) clinical trial (dose-response study) with 24 participating facilities throughout Japan to investigate the efficacy and safety of FGF-2 using placebo and the investigational drug containing $0.2 \%, 0.3 \%$, and $0.4 \% \mathrm{FGF}-2$ (2005-2007). The results showed that all investigational drugs containing FGF-2 induced statistically significant new alveolar bone formation, and $0.3 \%$ FGF-2 was the clinically recommended dose [6] (Fig. 12.2a). Based on these results, we then implemented a phase III (PIII) clinical study (randomized double-blind parallelgroup comparison study) with 23 participating facilities throughout Japan to investigate the efficacy and safety of FGF-2 using placebo and the investigational drug containing $0.3 \%$ FGF-2 (2008-2010). The results confirmed that the investigational drug containing $0.3 \%$ FGF-2 significantly increased alveolar bone $(p<0.001)$ (Fig. 12.2b) [7]. Additionally, no cases throughout the entire clinical study period were problematic in terms of safety.

We conducted detailed investigations into the effect of FGF-2 on cultured human periodontal ligament-derived cells to elucidate the mechanism of FGF-2-induced periodontal tissue regeneration; we predict the action mechanism to be as follows (Fig. 12.3). First, during the initial stages of wound healing, FGF-2 promotes the proliferation of stem cells while retaining their undifferentiated state and increases 


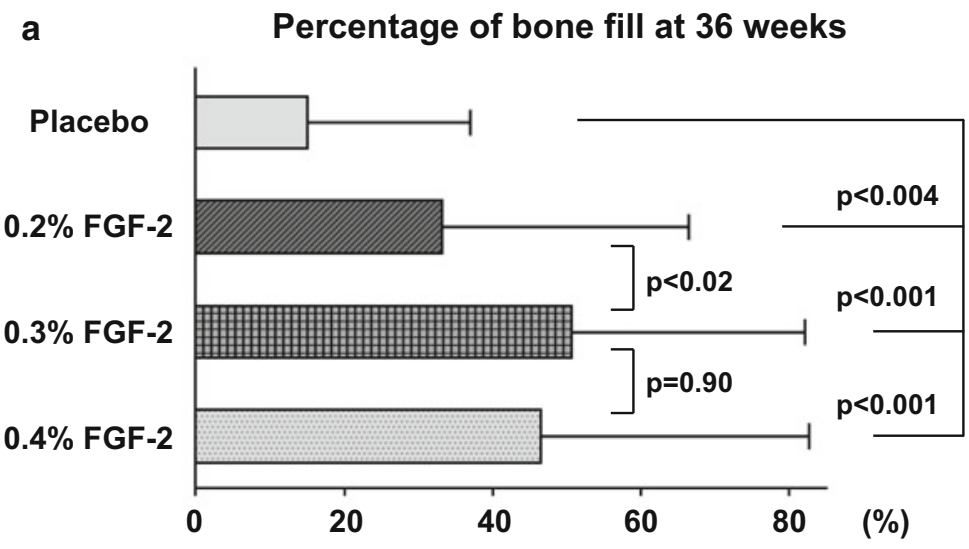

b Percentage of bone fill at 36 weeks

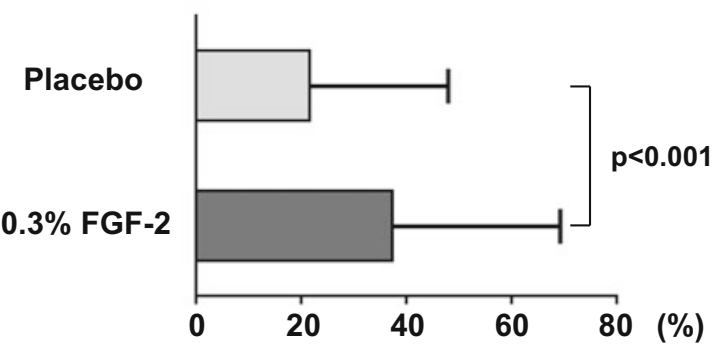

Fig. 12.2 Rate of increase in the height of alveolar bone 9 months after FGF-2 administration (clinical trial results on efficacy). (a) Late phase II clinical study: The subjects were divided into four groups (placebo group and $0.2 \%, 0.3 \%$, and $0.4 \%$ FGF-2 treatment groups); each investigational drug was administered under double-blinded conditions. The figure shows the digitized results of a standardized dental $\mathrm{x}$-ray taken 9 months after administration of the investigational drug and shows the percentage of the depth of the intrabony defect prior to treatment that had been filled with new bone (Adapted from literature [6]). (b) Phase III clinical study: The subjects were divided into two groups (placebo group and 0.3\% FGF-2 treatment group); each investigational drug was administered under double-blinded conditions. The figure shows the digitized results of a standardized dental $x$-ray taken 9 months after administration of the investigational drug and shows the percentage of the depth of the intrabony defect compared to before treatment that had been filled with new bone (Adapted from literature [7])

the cell density of periodontal tissue stem cells at the wound site. Furthermore, FGF-2 promotes angiogenesis and the production of specific extracellular matrix molecules, creating a suitable local environment for the regeneration of periodontal tissue. After the effect of the locally administered FGF-2 is removed from the administration site through degradation or other actions, the periodontal tissue stem cells, which had increased in number, begin to differentiate into hard tissue-forming cells in the optimal environment created by FGF-2 administration. This promotes 


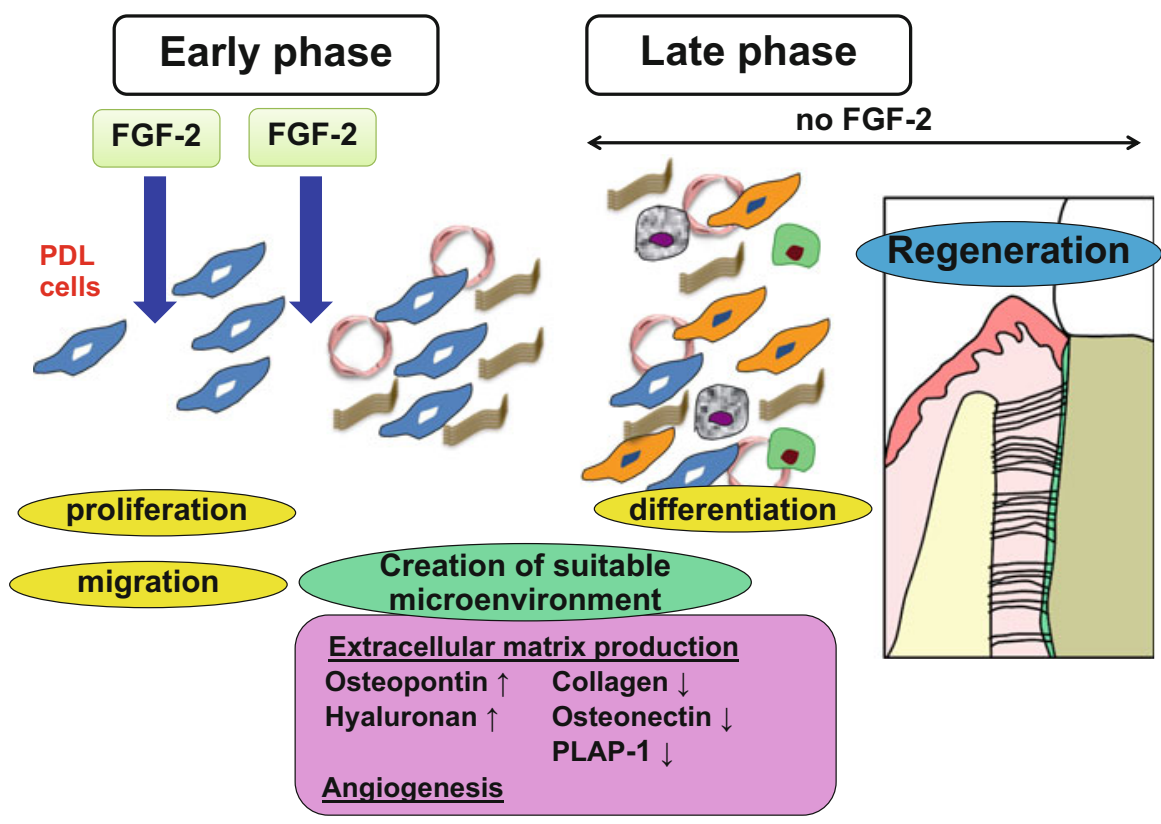

Fig. 12.3 Mechanism of induction of periodontal tissue regeneration with FGF-2. FGF-2 strongly promotes the migration and proliferation of immature human periodontal ligament (PDL) cells while retaining the cells' differentiation potential and increases the number of periodontal tissue stem cells at the site of periodontal tissue loss. Furthermore, FGF-2 promotes the production of angiogenesis and various extracellular matrices at the administration site, creating a suitable local environment for "periodontal tissue regeneration"

the regeneration of periodontal tissue, both in number and time period, including the formation of new alveolar bone and cementum [8].

\subsection{Future Outlook of Cytokine Therapy Using FGF-2}

The concept of "scaffold" has not been introduced for FGF-2 preparations, and clinical studies are currently underway. This is because the aim of both clinical studies was to first clarify the efficacy and safety of FGF-2 alone as a drug. However, in the next stage, we will examine tissue engineering-type methods for the FGF-2 carrier.

To date, in animal experiments using a severe periodontal tissue loss model (one wall intrabony defect model), we have found that following administration of $0.3 \%$ FGF-2 with $\beta$-TCP, there is no recession of the gingiva postoperatively and that significant periodontal tissue regeneration is induced at the same loss site [9]. If a new carrier for FGF-2 preparation is developed that can retain the space (spacemaking) at which the regeneration of periodontal tissue is expected, and with the 
appropriate formativeness and osteoconductive properties required by operators, the application of FGF-2 preparations is expected to be further broadened. Additionally, the results of animal experiments suggest that if the $0.3 \%$ FGF-2 preparation is used concurrently during dental implant surgery, the healing time to achieve osseointegration will be shortened and robust integration will be achieved; further verification of these concepts is required in the future [10].

\subsection{Possibility of Periodontal Tissue Regenerative Therapy with Stem Cell Transplantation}

The periodontal tissue regenerative therapy described above promotes the regeneration of periodontal tissue by inducing the functions of stem cells present in all periodontal ligaments. However, the number of stem cells in periodontal ligament decreases with age [11]. Therefore, in elderly people and in cases of severe periodontal disease, we do not expect an adequate amount of regeneration simply by activating the stem cells present in the periodontal ligament. In these cases, it may be necessary to promote periodontal tissue regeneration by transplanting mesenchymal stem cells collected from other tissue in the same patient to the site of periodontal tissue loss.

The use of induced pluripotent stem cells is expected in the future, but it will still be some time before this technology can be clinically applied in the field of dentistry. However, it has been clarified that undifferentiated mesenchymal stem cells are present in a variety of tissues in our bodies, even after reaching adulthood. Studies are currently being conducted to regenerate periodontal tissue by transplanting stem cells collected from these tissues into the site of periodontal tissue loss together with suitable scaffolding materials.

Examples of bone marrow cells used to regenerate periodontal tissue are as follows. There is a report showing that mixing bone marrow cells collected from the ilium or other such sites with platelet-rich plasma and transplanting the mixture into the site of periodontal tissue loss is effective for inducing periodontal tissue regeneration [12], whereas another report found that after stimulating and proliferating collected bone marrow cells with FGF-2 in the laboratory, subsequent mixing the cells with collagen gel as scaffolding material promotes periodontal tissue regeneration [13]. In these cases, undifferentiated mesenchymal cells and osteoblast progenitor cells in the bone marrow were used as stem cell sources during periodontal tissue regeneration.

Another study investigated the promotion of periodontal tissue regeneration by collecting tissue slices from the maxilla periosteum, culturing the cells collected from the same tissue slice (periosteum-derived cells: expected to be cells with high osteogenic potential) in a sheet format, and transplanting the cultured cells together with platelet-rich plasma and hydroxyapatite into the site of periodontal tissue loss; this process stimulated new alveolar bone formation and promoted regeneration 


\section{Periodontal regeneration by transplantation of ADMPC}

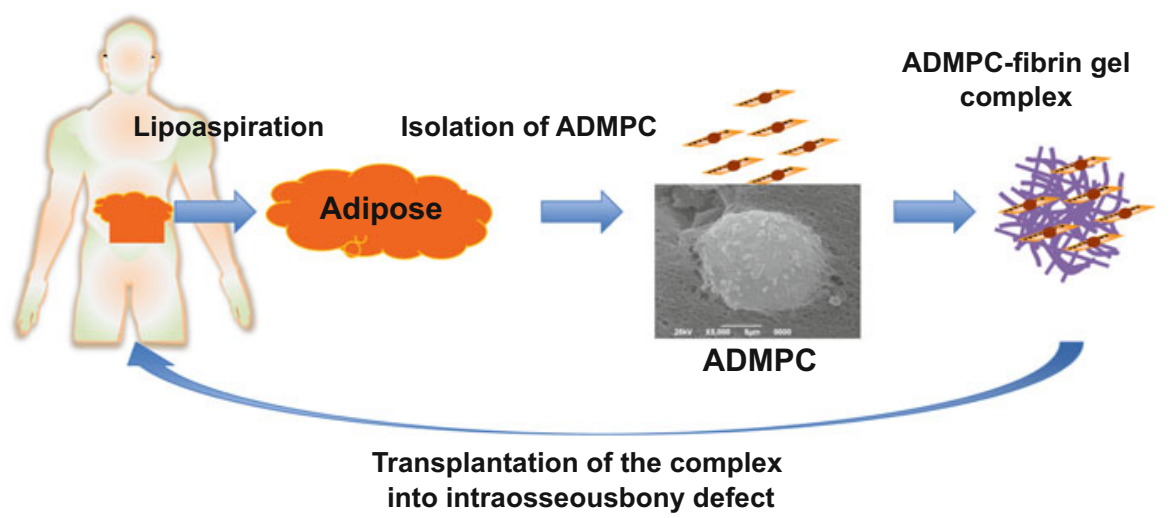

Fig. 12.4 Periodontal regeneration by transplantation of ADMPC. Approximately $30 \mathrm{~mL}$ of abdominal adipose tissue was suction sampled from the patient with periodontal disease, and ADMPCs were collected from the same tissue in the Cell Processing Center. These cells were then cultured until a sufficient volume of ADMPCs was acquired, which was then mixed with fibrin gel. Regeneration of periodontal tissue was induced by transplanting the same admixture into the site of the intrabony defect during periodontal surgery in the same patient

[14]. Furthermore, another study investigated culturing periodontal ligament cells in a sheet format, transplanting the cells onto the surface of a tooth root that had been exposed at the site of periodontal tissue loss, and transplanting $\beta$-TCP at the site of bone loss for total regeneration of periodontal tissue [15].

In our laboratory, we focused on adipose tissue, which we consider to be less of a burden on the patient during collection and a safer tissue. We investigated the use of undifferentiated mesenchymal stem cells present in another tissue as a source of stem cells (Fig. 12.4). We previously reported that adipose tissue-derived multilineage progenitor cells (ADMPCs) collected using our method differentiate into osteoblasts, myocardial cells, liver cells, and insulin-producing cells, as ADMPCs are pluripotent [16-19]. We also confirmed in in vitro that ADMPC isolated from human adipose tissue can differentiate into osteoblasts and periodontal ligament cells. Next, we investigated the effect of inducing periodontal tissue regeneration by ADMPC transplantation in a beagle periodontal disease model. We created artificial periodontal tissue loss (class II furcation involvement) at the furcation site of the fourth premolars in beagles and transplanted ADMPC isolated from adipose tissue collected from the abdominal area together with fibrin gel into the same site of the defect. The results confirmed significant regeneration of periodontal tissue at the transplantation site [20]. Interestingly, ADMPC had a remarkable trophic effect; insulin-like growth factor binding protein 6, which was secreted from ADMPC in large quantities, promoted the differentiation of periodontal tissue stem cells [21]. These results strongly suggest that adipose tissue, which is abundant in the body and 
can be easily and safely collected, can be used as a source of stem cells to promote the regeneration of periodontal tissue.

\subsection{Conclusion}

Periodontal tissue is a complex tissue comprised of alveolar bone, cementum, periodontal ligament, and gingiva, and the end target is to regenerate each type of tissue at will depending on the extent of periodontal tissue destruction. In the future, after ensuring the safety of this therapy, optimal scaffolding material can be customized for periodontal tissue regeneration to support stem cell migration and proliferation, as well as differentiation into osteoblasts and cementoblasts. The application of cytokine therapy using FGF-2 and periodontal tissue regenerative therapy through stem cell transplantation of ADMPC will not be restricted to the field of dentistry, but can also be expanded to other medical fields. Additionally, in the near future, we hope to establish patient-specific periodontal tissue regenerative therapy by combining optimal conditions with cytokines, stem cells, and scaffolding materials.

\section{References}

1. Seo BM, Miura M, Gronthos S, Bartold PM, Batouli S, Brahim J, et al. Investigation of multipotent postnatal stem cells from human periodontal ligament. Lancet. 2004;364:149-55.

2. Beertsen W, McCulloch CA, Sodek J. The periodontal ligament: a unique, multifunctional connective tissue. Periodontology. 1997;13:20-40.

3. Takayama S, Murakami S, Shimabukuro Y, Kitamura M, Okada H. Periodontal regeneration by FGF-2 (bFGF) in primate models. J Dent Res. 2001;81:2075-9.

4. Murakami S, Takayama S, Kitamura M, Shimabukuro Y, Yanagi K, Ikezawa K, et al. Recombinant human basic fibroblast growth factor (bFGF) stimulates periodontal regeneration in class II furcation defects created in beagle dogs. J Periodontal Res. 2003;38:97-103.

5. Kitamura M, Nakashima K, Kowashi Y, Fujii T, Shimauchi H, Sasano T, et al. Periodontal tissue regeneration using fibroblast growth factor-2 periodontal tissue regeneration using fibroblast growth factor-2: randomized controlled phase II clinical trial. PLoS One. 2008;3:e2611.

6. Kitamura M, Akamatsu M, Machigashira M, Hara Y, Sakagami R, Hirofuji T, et al. FGF-2 stimulates periodontal regeneration: results of a multi-center randomized clinical trial. J Dent Res. 2011;90:35-40.

7. Kitamura M, Akamatsu M, Kawanami M, Furuichi Y, Fujii T, Mori M, et al. Randomized placebo-controlled and controlled non-inferiority phase III trials comparing trafermin, a recombinant human fibroblast growth factor 2, and enamel matrix derivative in periodontal regeneration in intrabony defects. J Bone Miner Res. 2016;31:80.

8. Murakami S. Periodontal tissue regeneration by signalling molecule(s): what role does basic fibroblast growth factor (FGF-2) have in periodontal therapy? Periodontol 2000. 2011;56:188-208.

9. Anzai J, Kitamura M, Nozaki T, Nagayasu T, Terashima A, Asano T, et al. Effects of concomitant use of fibroblast growth factor (FGF)-2 with beta-tricalcium phosphate ( $\beta$-TCP) on the beagle dog 1-wall periodontal defect model. Biochem Biophys Res Commun. 2010;403:345-50. 
10. Nagayasu-Tanaka T, Nozaki T, Anzai J, Noriko S, Terashima A, Miki K, et al. FGF-2 promotes initial osseointegration and enhances stability of implants with low primary stability. Clin Oral Implants Res. 2016; in press.

11. Zheng W, Wang S, Ma D, Tang L, Duan Y, Jin Y. Loss of proliferation and differentiation capacity of aged human periodontal ligament stem cells and rejuvenation by exposure to the young extrinsic environment. Tissue Eng A. 2009;15:2363-71.

12. Yamada Y, Ueda M, Hibi H, Baba S. A novel approach to periodontal tissue regeneration with mesenchymal stem cells (MSCs) and platelet-rich plasma (PRP) using tissue engineering technology: a clinical case report. Int J Periodontics Restor Dent. 2006;26:363-9.

13. Kawaguchi H, Kurihara H. Clinical trial of periodontal tissue regeneration. Nippon Rinsho. 2008;66:948-54.

14. Okuda K, Yamamiya K, Kawase T, Mizuno H, Ueda M, Yoshie H. Treatment of human infrabony periodontal defects by grafting human cultured periosteum sheets combined with platelet-rich plasma and porous hydroxyapatite granules: case series. Int Acad Periodontol. 2009;11:206-13.

15. Iwata T, Yamato M, Tsuchioka H, Takagi R, Mukobata S, Washio K, et al. Periodontal regeneration with multi-layered periodontal ligament-derived cell sheets in a canine model. Biomaterials. 2009;30:2716-23.

16. Okura H, Komoda H, Fumimoto Y, Lee CM, Nishida T, Sawa Y, et al. Transdifferentiation of human adipose tissue-derived stromal cells into insulin-producing clusters. J Artif Organs. 2009; 12:123-30.

17. Okura H, Matsuyama A, Lee CM, Saga A, Kakuta-Yamamoto A, Nagao A, et al. Cardiomyoblast-like cells differentiated from human adipose tissue-derived mesenchymal stem cells improve left ventricular dysfunction and survival in a rat myocardial infarction model. Tissue Eng C Methods. 2010;16:417-25.

18. Okura H, Komoda H, Saga A, Kakuta-Yamamoto A, Hamada Y, Fumimoto Y, et al. A properties of hepatocyte-like cell clusters from human adipose tissue-derived mesenchymal stem cells. Tissue Eng C Methods. 2010;16:761-70.

19. Komoda H, Okura H, Lee CM, Sougawa N, Iwayama T, Hashikawa T, et al. Reduction of $\mathrm{N}$-glycolylneuraminic acid xenoantigen on human adipose tissue-derived stromal cells/mesenchymal stem cells leads to safer and more useful cell sources for various stem cell therapies. Tissue Eng A. 2010;16:1143-55.

20. Ozasa M, Sawada K, Iwayama T, Yamamoto S, Morimoto C, Okura H, et al. Periodontal tissue regeneration by transplantation of adipose tissue-derived multi-lineage progenitor cells. Inflamm Regen. 2014;34:109-16.

21. Sawada K, Takedachi M, Yamamoto S, Morimoto C, Ozasa M, Iwayama T, et al. Trophic factors from adipose tissue-derived multi-lineage progenitor cells promote cytodifferentiation of periodontal ligament cells. Biochem Biophys Res Commun. 2015;464:299-305.

Open Access This chapter is distributed under the terms of the Creative Commons Attribution 4.0 International License (http://creativecommons.org/licenses/by/4.0/), which permits use, duplication, adaptation, distribution and reproduction in any medium or format, as long as you give appropriate credit to the original author(s) and the source, provide a link to the Creative Commons license and indicate if changes were made.

The images or other third party material in this chapter are included in the work's Creative Commons license, unless indicated otherwise in the credit line; if such material is not included in the work's Creative Commons license and the respective action is not permitted by statutory regulation, users will need to obtain permission from the license holder to duplicate, adapt or reproduce the material.

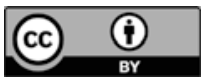

